\title{
Methods for Converging Correlation Energies within the Dielectric Matrix Formalism
}

\author{
Anant Dixit, $1, *$ Julien Claudot, ${ }^{1}$ Tim Gould, ${ }^{2}$ Sébastien Lebègue, ${ }^{1,3}$ and Dario Rocca ${ }^{1,3,+\dagger}$ \\ ${ }^{1}$ Université de Lorraine, LPCT, UMR 7019, \\ 54506 Vandouvre-lès-Nancy, France \\ ${ }^{2}$ Qld Micro- and Nanotechnology Centre, \\ Griffith University, Nathan, Qld 4111, Australia \\ ${ }^{3}$ CNRS, LPCT, UMR 7019, 54506 Vandouvre-lès-Nancy, France
}

(Dated: February 12, 2018)

\begin{abstract}
Within the dielectric matrix formalism, the random phase approximation (RPA) and analogous methods that include exchange effects are promising approaches to overcome some of the limitations of traditional density functional theory approximations. The RPA-type methods however have a significantly higher computational cost, and, similarly to correlated quantum chemical methods, are characterized by a slow basis set convergence. In this work we analyzed two different schemes to converge the correlation energy, one based on a more traditional complete basis set (CBS) extrapolation and one that converges energy differences by accounting for the size-consistency property. These two approaches have been systematically tested on the A24 test set, for six points on the potential energy surface of the methane-formaldehyde complex, and for reaction energies involving the breaking and formation of covalent bonds. While both methods converge to similar results at similar rates, the computation of size-consistent energy differences has the advantage of not relying on the choice of a specific extrapolation model.
\end{abstract}




\section{INTRODUCTION}

Density functional theory (DFT) is one of the most widely used approaches in computational materials science ${ }^{-}$. The Kohn-Sham formalism is computationally efficient and allows one to obtain the ground state energy, whose accuracy depends on the approximation of the exchange-correlation (xc) functional ${ }^{2}$. The most common approximations are the local density $^{\underline{3}}$ (LDA) and generalized gradient approximations $\underline{\underline{4}}^{\underline{4}}$ (GGA), wherein the xc functional depends on the density and the gradient of the density. In spite of the success of these functionals, they do not usually reach chemical accuracy and lack a proper description of the long-range van der Waals (vdW) forces.

The adiabatic connection fluctuation dissipation theorem (ACFDT) provides a practical framework to develop approximations for DFT functionals $s^{5} \underline{6}$. Specifically, this approach expresses the xc contribution in terms of the response functions (polarizabilities or, equivalently, dielectric matrices). While exact in principle, the ACFDT requires approximations in practice, as the interacting polarizability is unknown.

The random phase approximation (RPA) is the most simple approximation used within the ACFDT. Though relatively basic in form, the RPA xc functional has a high level of accuracy and correctly describes the long-range behavior of the vdW forces. This has been demonstrated by applications to a variety of different types of systems such as molecules ${ }^{7-16}$, layered materials $17-\underline{20}$, solids $\underline{21}-\underline{25}$, and molecules adsorbed on surfaces $\underline{26}$. Nevertheless the RPA suffers from some limitations, as correlation energies are sizeably overestimated 27 , and interaction energies tend to be underestimated ${ }^{28}$. Several schemes have been proposed to

improve the RPA with varying degrees of success $8,10,27,29-39$. All these schemes, including RPA, are seamless in the sense that they treat physics at all ranges (short, intermediate, long) using the same fundamental physics. They can thus smoothly capture disparate processes such as bond-breaking and dispersion forces. The latter are treated to all orders in manybody interactions.

In this work we will focus on two recently proposed methods - the electron-hole timedependent Hartree-Fock (eh-TDHF) and the adiabatic connection second-order screened exchange (AC-SOSEX) $\underline{14} \underline{-16}$. These two approaches, referred to as the beyond-RPA methods henceforth, improve the RPA through the introduction of exchange effects absent in the RPA. Their accuracy was demonstrated by computing chemical reaction energies involving the 
breaking and formation of covalent bonds 14,15 and interaction energies for weakly interacting systems (A24 and S22 test sets) $\frac{16}{2}$. As shown by these applications, the interest in RPAtype methods is not limited to $\mathrm{vdW}$ interactions, where they compete with empirically corrected $\underline{40-42}$ and non-local $\underline{43,44}$ functionals, but includes also other types of bonding.

Compared to semi-local or hybrid functionals, the ACFDT approaches come at a comparatively high computational cost, although these are typically cheaper than the most sophisticated wavefunction methods. Additionally, the RPA and beyond-RPA correlation energies are characterized by a slow basis set convergence. This behavior is analogous to that of traditional quantum chemical methods. Indeed, convergence of the correlation energy is known to be slow in correlated methods such as Møller-Plesset perturbation theory or coupled cluster theory $\underline{\underline{45}} \underline{\underline{52}}$. In order to overcome this issue, techniques to extrapolate the results to the complete basis set (CBS) limit are routinely used $\underline{47,50,53}$.

In this work we study the convergence of the RPA and beyond-RPA methods with respect to the basis set size. The numerical implementation used is based on the plane-wave (PW) basis set but the response functions are actually represented on an auxiliary basis set to rapidly converge the dielectric eigenvalues $\underline{13}, 15,16,54 \underline{58}$. Two approaches to converge the correlation energy are compared: (1) a more standard approach based on the CBS extrapolation $\frac{19,21,22}{2} ;(2)$ an alternative technique to rapidly converge energy differences by accounting for the size-consistency property $\underline{\underline{11}, 13,15,59}$. This second approach has never been discussed in detail in the literature. It is rarely applied in practical calculations, and unlike the CBS extrapolation, is not biased by the choice of a model function for the fitting. By computing several interaction energies of weakly bound molecular systems and reaction energies, we analyze the convergence and show that the two approaches lead to similar results.

In Section [1] we begin by summarizing briefly the RPA and beyond-RPA methods along with the numerical framework used in their implementation. Section【discuss the different techniques used to converge correlation energies. Numerical convergence tests for the A24 test set and the potential energy surface of the methane-formaldehyde system are presented in Section IV] Section V contains our conclusions. 


\section{THEORETICAL FRAMEWORK}

In this section, we summarize the theoretical framework for the RPA and beyond-RPA methods used in this work. A more complete discussion can be found in Refs. 14 and 15 . The three correlation energy approximations used are:

$$
\begin{aligned}
E_{c}^{\mathrm{RPA}} & =\frac{1}{2} \int_{-\infty}^{\infty} \frac{\mathrm{d} \omega}{2 \pi} \operatorname{Tr}\left[\ln \left\{\boldsymbol{I}-\chi^{0}(i \omega) \boldsymbol{K}\right\}+\chi^{0}(i \omega) \boldsymbol{K}\right] \\
E_{c}^{\mathrm{eh}-\mathrm{TDHF}} & =\frac{1}{2} \int_{-\infty}^{\infty} \frac{\mathrm{d} \omega}{2 \pi} \operatorname{Tr}\left[\ln \left\{\boldsymbol{I}-\chi^{0}(i \omega) \boldsymbol{B}\right\} \boldsymbol{B}^{-1} \boldsymbol{K}+\chi^{0}(i \omega) \boldsymbol{K}\right] \\
E_{c}^{\mathrm{AC}-S O S E X} & =\frac{1}{2} \int_{-\infty}^{\infty} \frac{\mathrm{d} \omega}{2 \pi} \operatorname{Tr}\left[\ln \left\{\boldsymbol{I}-\chi^{0}(i \omega) \boldsymbol{K}\right\} \boldsymbol{K}^{-1} \boldsymbol{B}+\chi^{0}(i \omega) \boldsymbol{B}\right],
\end{aligned}
$$

where $E_{c}^{\mathrm{RPA}}$ is the RPA correlation energy, $E_{c}^{\mathrm{eh}-\mathrm{TDHF}}$ is the electron-hole time-dependent Hartree-Fock (eh-TDHF) correlation energy, and $E_{c}^{\text {AC-SOSEX }}$ is the adiabatic connection second-order screened-exchange (AC-SOSEX) correlation energy. In Eqs. 1 3 w is the frequency, $\boldsymbol{I}$ denotes the identity matrix, and $\boldsymbol{K}$ is the 'direct' kernel, whose matrix elements are defined as,

$$
K_{v c, v^{\prime} c^{\prime}}=2\left\langle\phi_{v} \phi_{c}|V| \phi_{v^{\prime}} \phi_{c^{\prime}}\right\rangle=2 \int \mathrm{d} \boldsymbol{r} \mathrm{d} \boldsymbol{r}^{\prime} \phi_{v}(\boldsymbol{r}) \phi_{c}(\boldsymbol{r}) V\left(\boldsymbol{r}, \boldsymbol{r}^{\prime}\right) \phi_{v^{\prime}}\left(\boldsymbol{r}^{\prime}\right) \phi_{c^{\prime}}\left(\boldsymbol{r}^{\prime}\right)
$$

The $\boldsymbol{B}$ matrix is an approximate exchange kernel containing only electron-hole products, whose matrix elements are defined as,

$$
B_{v c, v^{\prime} c^{\prime}}=2\left\langle\phi_{v} \phi_{c}|V| \phi_{v^{\prime}} \phi_{c^{\prime}}\right\rangle-\left\langle\phi_{v} \phi_{c^{\prime}}|V| \phi_{v^{\prime}} \phi_{c}\right\rangle
$$

In Eqs. 4 and 5, $v$ and $c$ denotes the valence (occupied) and conduction (unoccupied) states respectively, and $V\left(\boldsymbol{r}, \boldsymbol{r}^{\prime}\right)$ is the Coulomb potential. $\chi^{0}$ in Eqs. 1, 3 is the non-interacting response function, defined as,

$$
\chi_{v c, v^{\prime} c^{\prime}}^{0}(i \omega)=2 \Re\left(\frac{1}{i \omega-\varepsilon_{v}+\varepsilon_{c}}\right) \delta_{v v^{\prime}} \delta_{c c^{\prime}},
$$

with $\varepsilon_{v}$ and $\varepsilon_{c}$ denoting valence and conduction state energies, respectively, and $\Re$ is the real part. The term $\boldsymbol{I}-\chi^{0}(i \omega) \boldsymbol{K}$ in the correlation energy expressions is usually referred to as the dielectric matrix. 
Since Eq. 11 does not contain exchange effects, this expression of the RPA is sometimes referred to as the direct-RPA (d-RPA) in the quantum chemistry community. The approximations to the correlation energy in Eqs. 2 and 3are henceforth indicated as the beyond-RPA approximations, as they correct the RPA by including exchange effects through the matrix B.

The formalism presented here can be implemented in the PW basis set by using techniques to avoid the explicit calculation of conduction states 60 -63 response functions with a compact auxiliary basis set $\underline{13,15}, 16, \underline{55}-\underline{58,64}$. This auxiliary basis set can be efficiently obtained from an iterative diagonalization of a simplified polarizability containing the kinetic energy contribution only $\stackrel{13}{ }$, an approximation of the projective dielectric eigenpotential (PDEP) technique ${ }^{54}$. Further details can be found in Ref. 15, and the final working equations are summarized below. The response functions can then be represented with the kinetic-PDEP auxiliary basis set, whose $N_{\text {aux }}$ elements are denoted by $\left\{\Phi_{Q}\right\}$, as:

$$
\begin{aligned}
& C_{Q R}(i \omega)=4 \sum_{v}\left\langle\phi_{v}\left|\Phi_{Q} V^{1 / 2} \hat{P}_{c} \Re\left(i \omega+\epsilon_{v}-\hat{H}\right)^{-1} \hat{P}_{c} V^{1 / 2} \Phi_{R}\right| \phi_{v}\right\rangle \\
& J_{Q G}^{v v^{\prime}}(i \omega)=2\left\langle\phi_{v}\left|\Phi_{Q} V^{1 / 2} \hat{P}_{c} \Re\left(i \omega+\epsilon_{v}-\hat{H}\right)^{-1} \hat{P}_{c} V^{1 / 2} \Phi_{G}\right| \phi_{v^{\prime}}\right\rangle \\
& L_{G R}^{v v^{\prime}}(i \omega)=2\left\langle\phi_{v}\left|\Phi_{G} V^{1 / 2} \hat{P}_{c} \Re\left(i \omega+\epsilon_{v^{\prime}}-\hat{H}\right)^{-1} \hat{P}_{c} V^{1 / 2} \Phi_{R}\right| \phi_{v^{\prime}}\right\rangle \\
& Z_{Q R}(i \omega)=\sum_{v v^{\prime}} \sum_{G} J_{Q G}^{v v^{\prime}}(i \omega) L_{G R}^{v v^{\prime}}(i \omega),
\end{aligned}
$$

where $\hat{H}$ is the ground-state Kohn-Sham Hamiltonian and $\hat{P}_{c}=\sum_{c}\left|\phi_{c}\right\rangle\left\langle\phi_{c}\right|$ is the projector over the conduction state subspace. By using the completeness of the basis set this projector can be expressed as $\hat{P}_{c}=\hat{I}-\sum_{v}\left|\phi_{v}\right\rangle\left\langle\phi_{v}\right|$, which contains a sum over the occupied states only $\underline{60}$. From the definitions in Eqs. 77, 10, we can then reformulate Eqs. 1 13 as (see Ref. 15 for a complete derivation),

$$
E_{c}^{\mathrm{RPA}}=\frac{1}{2} \int_{-\infty}^{\infty} \frac{\mathrm{d} \omega}{2 \pi} \operatorname{Tr}[\ln \{\boldsymbol{I}-\boldsymbol{C}(i \omega)\}+\boldsymbol{C}(i \omega)]
$$

for the RPA,

$$
E_{c}^{\mathrm{eh}-\mathrm{TDHF}}=\frac{1}{2} \int_{-\infty}^{\infty} \frac{\mathrm{d} \omega}{2 \pi} \operatorname{Tr}\left[\ln \left\{\boldsymbol{I}-\boldsymbol{C}(i \omega)+\boldsymbol{Z}(i \omega) \boldsymbol{C}^{-1}(i \omega)\right\}\left\{\boldsymbol{C}(i \omega)-\boldsymbol{Z}(i \omega) \boldsymbol{C}^{-1}(i \omega)\right\}^{-1} \boldsymbol{C}(i \omega)+\boldsymbol{C}(i \omega)\right]
$$


for the eh-TDHF and

$$
E_{c}^{\mathrm{AC}-\operatorname{SoseX}}=\frac{1}{2} \int_{-\infty}^{\infty} \frac{\mathrm{d} \omega}{2 \pi} \operatorname{Tr}\left[(\ln \{\boldsymbol{I}-\boldsymbol{C}(i \omega)\}+\boldsymbol{C}(i \omega))\left(\boldsymbol{I}-\boldsymbol{C}^{-1}(i \omega) \boldsymbol{Z}(i \omega) \boldsymbol{C}^{-1}(i \omega)\right)\right]
$$

for the AC-SOSEX. An important advantage of this formalism is that the projector in the $\boldsymbol{C}$ and $\boldsymbol{Z}$ matrices implicitly includes all the conduction states within the PW basis. Additionally, the size of the $\boldsymbol{C}$ and $\boldsymbol{Z}$ matrices is reduced to $N_{\text {aux }} \times N_{\text {aux }}$, where $N_{\text {aux }} \ll N_{\text {eh }}$ ( $N_{e h}=N_{c} N_{v}$ being the number conduction-valence pairs), and $N_{a u x} \ll N_{P W}\left(N_{P W}\right.$ being the number of plane-waves). Finally, once the auxiliary basis set vectors $\left\{\Phi_{Q}\right\}$ are fixed, the frequency-dependent matrix elements in Eqs. 7,9 can be efficiently computed using the Lanczos algorithm developed previously 15,55,62,64. The methodology discussed in this section has been implemented within the Quantum Espresso package ${ }^{65.66}$, which uses plane-waves and pseudopotentials.

As with traditional correlated methods such as Møller-Plesset perturbation theory ${ }^{51}$ or coupled-cluster theory ${ }^{52}$ theory, the correlation energy expressions in Eqs. 1, 3 present a slow basis set convergence, due to difficulties in reproducing the Coulomb cusp. In Sec. III we analyze two different schemes to reach convergence, one based on energy differences and one on complete basis set extrapolation. Since the Møller-Plesset perturbation theory to second order (MP2) can also be reformulated in terms of response functions, and implemented in a similar numerical framework $\frac{67}{}$, the discussion below is valid for this approach as well.

\section{CONVERGENCE OF ENERGY DIFFERENCES VS. COMPLETE BASIS SET EXTRAPOLATION}

In our previous works $13,15,16,24$ we did not rely on any complete basis set extrapolation technique for the RPA or beyond-RPA methods, but we rather converged bare energy differences. This is a peculiar feature of our implementation that takes advantage of a compact auxiliary basis set which is designed to rapidly converge the eigenvalues of the response functions $\frac{13,54}{}$. In this work we give a detailed description of the methodology that we have been using so far, and compare it to a more traditional approach, the complete basis set extrapolation.

To understand how correlation energy differences are converged, a simple observation is necessary, illustrated by a simple example. Let us suppose that we are interested in 
computing the interaction energy of the argon dimer at the RPA level of theory $\underline{11}, \underline{59}$. We first consider the two atoms at a very large distance so that

$$
E^{\text {int }}=E^{\text {dimer }}-2 E^{\text {atom }} \approx 0
$$

where $E^{\text {int }}$ is the interaction energy, $E^{\text {dimer }}$ is the total dimer energy, and $E^{\text {atom }}$ is the total atom energy. Eq. 14 is a consequence of the size consistency of the RPA 68 . Clearly, each single contribution to the energy should be exactly compensated for, including the correlation energy: $E_{c}^{\text {int }}=E_{c}^{\text {dimer }}-2 E_{c}^{\text {atom }} \approx 0$. By using a single eigenvalue of the dielectric matrix for each frequency $\lambda_{1}(i \omega)$ (the largest in absolute value), the RPA correlation energy (Eq. 11) of each single argon atom is

$$
E_{c}^{a t o m}=\frac{1}{2} \int_{-\infty}^{\infty} \frac{\mathrm{d} \omega}{2 \pi}\left[\ln \left\{1-\lambda_{1}(i \omega)\right\}+\lambda_{1}(i \omega)\right]
$$

In order to obtain $E^{\text {int }} \approx 0$ it is now clear that the correlation energy of the dimer should be computed including two eigenvalues per frequency in the correlation energy expression:

$$
E_{c}^{\text {dimer }}=\frac{1}{2} \int_{-\infty}^{\infty} \frac{\mathrm{d} \omega}{2 \pi}\left[\ln \left\{1-\lambda_{1}(i \omega)\right\}+\lambda_{1}(i \omega)+\ln \left\{1-\lambda_{2}(i \omega)\right\}+\lambda_{2}(i \omega)\right]
$$

Indeed, since the interatomic distance is large, we have $\lambda_{2}(i \omega) \approx \lambda_{1}(i \omega)$. This error cancellation would have not been possible if we would have naively used the same number of eigenvalues for the atoms and the dimer. The same idea can be also applied to small interatomic distances; there should be a compensation between the eigenvalue contributions of the dimer and the monomers to induce error cancellation. Generally, at small or intermediate distances it is necessary to significantly increase the number of eigenvalues $N_{\text {eig }}$ included in the evaluation of the correlation energy to reach convergence, while maintaining the condition $N_{\text {eig }}^{\text {dimer }}=2 N_{\text {eig }}^{\text {monomer }}$. Henceforth, we refer to this approach as the size-consistent energy difference approach.

This idea can also be generalized to systems, whose components are different (e.g. a dimer of two different molecules), or which involve different types of interactions. When the PDEP technique was first introduced it was already understood that the number of eigenvalues necessary to reach a certain level of accuracy in the description of the response function is approximately proportional to the number of electrons $\underline{54}$. Accordingly, the number of eigenvalues (namely of auxiliary basis vectors) used to describe the response function should 
be chosen to be proportional to the number of electrons. For example, if in a certain molecular dimer, the monomer 1 has double the number of electrons than the monomer 2 , in the correlation energy equations we should always use $N_{\text {aux }}^{\text {monomer } 1}=2 N_{\text {aux }}^{\text {monomer } 2}$ and $N_{\text {aux }}^{\text {dimer }}=N_{\text {aux }}^{\text {monomer } 1}+N_{\text {aux }}^{\text {monomer } 2}=2 N_{\text {aux }}^{\text {monomer } 2}+N_{\text {aux }}^{\text {monomer } 2}$. As shown by the numerical results in Sec. IV this approach works well in practice.

All this discussion has been presented for the RPA. The eh-TDHF and AC-SOSEX correlation energies have a similar structure to the RPA (see Eqs. 20.3 or Eqs. 12, 13), but contain additional exchange terms. As shown in Eqs. 8,10, the additional exchange contributions have a structure similar to the response function in Eq. 7, the main difference being related to the $v \neq v^{\prime}$ terms. It was already observed that the PDEP basis set, specifically designed for the polarizability (Eq. 7), represents well also the exchange terms (Eqs. 8, 10] $)^{15,16}$. Because of these affinities between the RPA and the beyond-RPA methods discussed in this work, the same convergence techniques can be used in practical calculations for beyond-RPA methods as well.

By using the size-consistent energy difference technique, the interaction energy converges quite rapidly with respect to the auxiliary basis set size. As an example, we present in Fig. 1 the interaction energy of the formaldehyde dimer computed at the RPA and beyond-RPA levels of theory. The convergence with respect to $N_{\text {aux }}$ is quite rapid, with the beyond-RPA energies seeming to converge faster than RPA. Since the measurable physical observables are energy differences, convergence of the interaction energy with respect to the auxiliary basis set size is sufficient for practical applications. Empirically we have observed that $N_{\text {aux }}=40 \times N_{v}$ is sufficient to achieve convergence for most molecular systems, where $N_{v}$ is the number of Kohn-Sham states (half the number of valence electrons) in the system. This is a very small number compared to the number of plane-waves $\left(N_{P W}=163000\right.$ for the formaldehyde dimer), and convergence of the interaction energy can be achieved without resorting to extrapolation techniques. However, if we consider the correlation energies of the formaldehyde dimer and of the monomer separately, convergence is far from achieved. For example, by increasing the basis set from $35 \times N_{v}$ to $70 \times N_{v}$, the correlation energy decreases by about $10 \mathrm{kcal} / \mathrm{mol}$ for the dimer and by about $5 \mathrm{kcal} / \mathrm{mol}$ for the monomers; this leads to the error cancellation shown in Fig. 1,

It is important to mention that the size-consistent energy difference technique can in principle be used with different types of auxiliary basis sets. For example, plane-waves are 


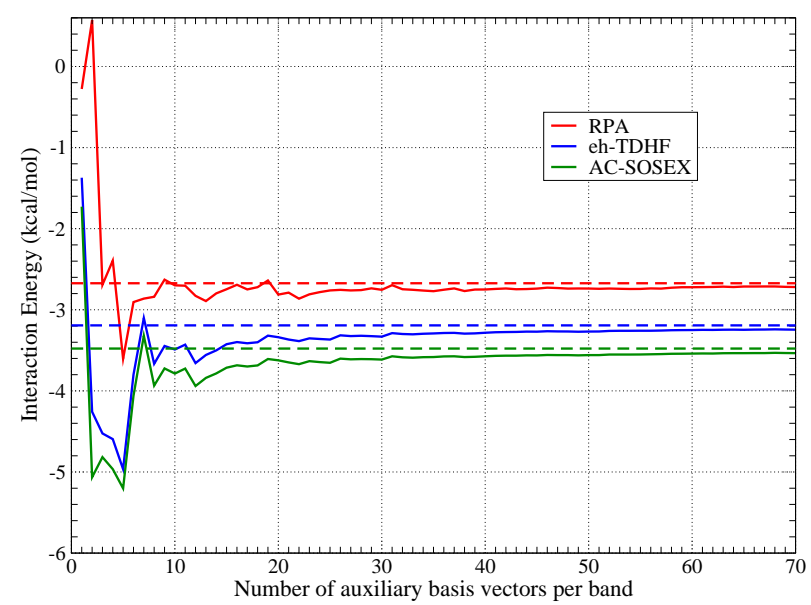

FIG. 1: Interaction energy (kcal/mol) of the formaldehyde dimer at the RPA (red), eh-TDHF (blue) and AC-SOSEX (green) levels of theory as a function of $N_{\text {aux }} / N_{v}$. Dashed lines indicate the CBS interaction energy values computed with Eq. 17.

traditionally used to represent dielectric matrices. For a given size of the dielectric matrix, the accuracy of the eigenvalues in the PW representation is lower than for the kinetic-PDEP auxiliary basis sets and, accordingly, also the energy differences less accurate. This implies that the use of PWs or other basis set, while compatible with the energy difference method, might lead to slower convergence.

The approach described in this section to converge energy differences has been previously used for homogeneous $11,13,59$ and heterogeneous systems $15,16,67$. In this work we will systematically study its accuracy for weakly bound systems and reaction energies involving the breaking and formation of covalent bonds (see Sec. IV]).

The complete basis set extrapolation scheme for the RPA and beyond-RPA correlation energies used in this work is,

$$
E_{c}^{N_{a u x}}=A+\frac{B}{N_{a u x}}
$$

where $A=E_{c}^{\infty}$ is the CBS correlation energy and $B$ is a system-dependent parameter. As shown in Fig. 2 for the formaldehyde dimer, the function in Eq. 17 provides a reasonable description of the behavior of the correlation energy as a function of $N_{\text {aux }}$. An analogous expression with an additional $C / N_{a u x}^{2}$ term was previously used to compute the interaction energy of molecular crystals 22 . The higher order contribution can be discarded for sufficiently high values of $N_{\text {aux }}$ (this is the case for the results of Sec. IV]). In Ref. 22, the PDEP method was used to exactly diagonalize the dielectric matrix for each different value of the frequency. 


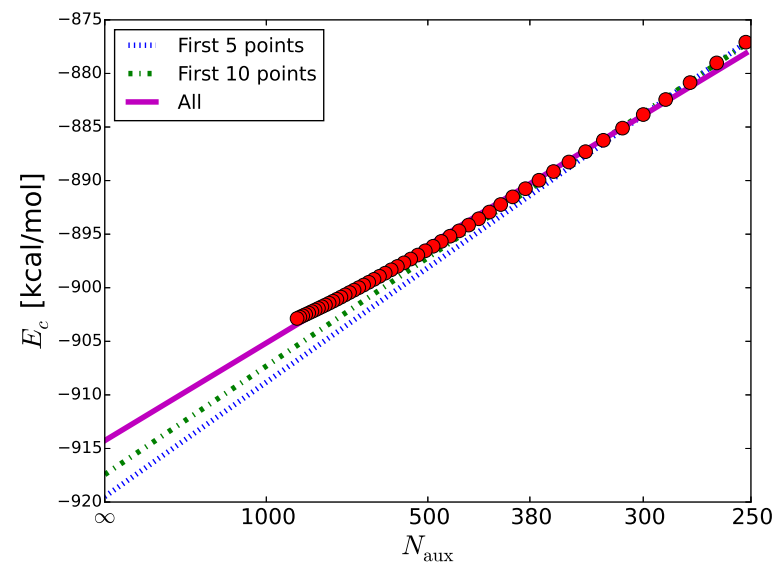

FIG. 2: Correlation energy (red dots) of the formaldehyde dimer at the RPA level of theory as a function of $1 / N_{\text {aux }}$. The points correspond to the range from $N_{a u x}=21 \times N_{v}$ to $N_{a u x}=70 \times N_{v}$. The continous line denotes a linear fit of the data. The dotted and dot-dash lines show partial fits, demonstrating how rapidly the approach converges.

By comparison our approach is approximated, as the basis set generated in the static case is used in the dynamical case as well.

Beyond working well in practice, Eq. 17 is similar to the function routinely used in the quantum chemistry community to extrapolate the correlation energy of approximations based on Møller-Plesset perturbation theory and coupled cluster theory. In these approaches the correlation energy is extrapolated to the infinite basis set limit as $\underline{45}-\underline{48}$,

$$
E_{c}^{X}=E_{c}^{\infty}+\frac{B}{X^{3}}
$$

with $X$ denoting the cardinal number of the basis set, $E_{c}^{\infty}$ being its "true" correlation energy evaluated at the complete basis set limit, and $B$ being a system-, and method-dependent constant. The number of the basis set functions is typically

$$
N_{X}=\frac{1}{3}(X+1)\left(X+\frac{3}{2}\right)(X+2),
$$

namely $N_{X} \propto X^{3}$, and for sufficiently large values of $N_{X}$ we get to leading order

$$
E_{c}^{X}=E_{c}^{\infty}+\frac{B^{\prime}}{N_{X}}
$$

which has a form similar to Eq. 17. As with $B$ above, $B^{\prime}$ depends on the system and method. 
Alternative extrapolation techniques used in the quantum chemistry community include expressions with variable exponents such as $\underline{49.50}$,

$$
E_{c}^{X}=E_{c}^{\infty}+\frac{B}{X^{C}},
$$

where, typically, small deviations are observed from $C=3$ (Eq. 18).

In standard implementations using plane-wave basis sets, the RPA correlation energy is often extrapolated to CBS as $\underline{21}$,

$$
E_{c}^{|\boldsymbol{G}|_{c u t}}=E_{c}^{\infty}+\frac{B}{\left|\boldsymbol{G}_{c u t}\right|^{3}}=E_{c}^{\infty}+\frac{B^{\prime}}{N_{P W}},
$$

where $\left|\boldsymbol{G}_{\text {cut }}\right|$ is the maximum reciprocal lattice vector used to represent the response function and $N_{P W}$ is the number of plane-waves. This is different with respect to Eq. 20, where $N_{X}$ is the basis set used to describe the full electronic structure and not just the dielectric matrices (which are not even used in typical coupled-cluster or Møller-Plesset perturbation theory formulations). Our implementation is also based on a plane-wave basis set but the response functions are represented on a system-dependent optimized basis set. Because of this difference, the range of values of $N_{\text {aux }}$ used to apply the extrapolation function in Eq. 17 might be different from the range of values of $N_{P W}$ used for the extrapolation function in Eq. 22. Since the basic numerical framework is the same, the two implementations should give indistinguishable results in the CBS limit.

To complete the literature review, we mention that Björkman et al $\underline{\underline{19}}$ and Gulans $\underline{20}$ used a different approach to converge RPA results for layered materials like hexagonal boron nitride and molybdenum di-sulphide. In these works it was observed that the correlation energy differences of layered materials converge as $\left|\boldsymbol{G}_{c u t}\right|^{-5}$, and this observation was used to obtain CBS results. By using this approach it was possible to obtain accurate CBS interaction energies with much lower kinetic energy cut-offs $(\sim 100-150 \mathrm{eV})$. However, it is not clear if this methodology is valid in general for different classes of materials, or is a specific property of spatially infinite planar solids. Though attractive, such behavior of correlation energy differences was not observed in our calculations.

Both approaches based on the CBS extrapolation and on the size-consistent energy differences present advantages and disadvantages. In the case of the CBS extrapolation, the choice of the model is to a certain extent arbitrary (for example, Eq. 18 or Eq. 21); additionally, the final result might be affected by small fluctuations in the correlation energies 
used for the fitting. On the plus side this approach can be applied to any kind of system. By contrast, the alternative technique proposed here is not biased by the model chosen, but is based on reasonable assumptions about size-consistency. However, this approach might suffer from slow convergence when energy differences are computed between systems that have a very different dielectric eigenvalue structure.

Let us consider an extreme example: the interaction between a small molecule (monomer 1 ), with only one occupied state, and a larger molecule (monomer 2 ), with nine occupied states. Specifically, we suppose that $\lambda_{400}^{\text {monomer } 2}(i \omega)>\lambda_{1}^{\text {monomer } 1}(i \omega)$, where $\lambda_{i}(i \omega)$ indicates the $i^{\text {th }}$ dielectric eigenvalue for each system. If we compute the RPA correlation energy for the corresponding dimer when the two molecules are separated by a large distance, by using just 400 eigenvalues $\left(40 \times N_{v}\right)$, we would have,

$$
\begin{aligned}
E_{c}^{\text {dimer }} & =\frac{1}{2} \int_{-\infty}^{\infty} \frac{\mathrm{d} \omega}{2 \pi} \sum_{i}^{400}\left[\ln \left\{1-\lambda_{i}^{\text {dimer }}(i \omega)\right\}+\lambda_{i}^{\text {dimer }}(i \omega)\right] \\
& =\frac{1}{2} \int_{-\infty}^{\infty} \frac{\mathrm{d} \omega}{2 \pi} \sum_{i}^{400}\left[\ln \left\{1-\lambda_{i}^{\text {monomer } 1}(i \omega)\right\}+\lambda_{i}^{\text {monomer } 1}(i \omega)\right]
\end{aligned}
$$

it is clear that in this case, the energy of the dimer is fully dominated by the eigenvalues of the larger system. In other words, there are no eigenvalues of monomer 2 present in the eigenvalue listing of the dimer, and no error cancellation can be obtained for $N_{a u x}=40 \times N_{v}$. Accordingly, convergence can be achieved only for very large values of $N_{a u x}$. This slow convergence could also appear in cases where the correlation energy difference is computed between two systems and a corresponding complex containing the same atoms rearranged in a significantly different structure with a significantly different dielectric eigenvalues. In this context the reaction energy test set discussed in Sec. IV.C could represent a problematic example, as it contains reactants and products with different molecular structures and possibly significantly different dielectric spectrum; still the size-consistent energy difference method provides accurate estimates of the reaction energies. In general, the size-consistent energy difference approach allows for a systematic study of convergence as a function of $N_{\text {aux }}$, and problematic cases can be identified and potentially dealt with, a significant advantage over the the CBS technique.

In the following section, we present the RPA and beyond-RPA interaction energy results for the A24 test set $\underline{49}$, for the methane-formaldehyde complex (MFC), and for reaction 
energies as computed for different auxiliary basis set sizes $\left(N_{\text {aux }}\right)$ with and without the extrapolation technique of Eq. 17.

\section{NUMERICAL RESULTS AND DISCUSSION}

\section{A. A24 Test Set}

To verify the energy differences, and compare the values with those obtained with the CBS extrapolation technique, we start by considering the A24 test set proposed by Řezáč et al., which provides interaction energies for 24 molecular dimers of three types - hydrogenbonded, dispersion-dominated and mixed-type ${ }^{49}$. A comparison of the RPA and beyondRPA methods with the reference CCSDT $(\mathrm{Q})$ values has been published in Ref. 16, and is not repeated here. As in previous work, numerical results are based on the FHI pseudopotentials available on the Quantum Espresso online library. In the case of weakly bound systems in was shown that the pseudopotentials have little influence on the final results $\frac{15,16}{1}$. In this work, we focus on estimating the inaccuracies due to the use of an incomplete auxiliary basis set; as the molecules included in the A24 are relatively small, a systematic study is possible by increasing $N_{a u x}$ to large values. For establishing a reference, the RPA and beyond-RPA calculations were first performed using an auxiliary basis set size of $70 \times N_{v}$ (in Ref. 16 this number was limited to $40 \times N_{v}$ for most systems) and then the absolute values of the correlation energies were extrapolated to CBS using Eq. 17 with 30 points from $41 \times N_{v}$ to $70 \times N_{v}$. Although small numerical fluctuations might still be present, this procedure, by including a large number of points in the fitting and a large $N_{\text {aux }}$, provides reasonably accurate reference values to study the convergence of RPA and beyond-RPA methods.

The choice of the $41 N_{v}-70 N_{v}$ CBS value as a reference will also be used for the other numerical examples considered in the following subsections. As we will consider below values from size-consistent energy differences and CBS extrapolations from different intervals of $N_{\text {aux }}$, it is important to keep in mind that our specific choice of the reference introduces a bias in favor of CBS results, which in general will be characterized by a more regular and predictable convergence.

In the following we study the level of convergence of CBS values obtained by extrapolating 20 points in three different ranges: $N_{a u x} / N_{v}=11-30,21-40$, and 31-50. We compare these 


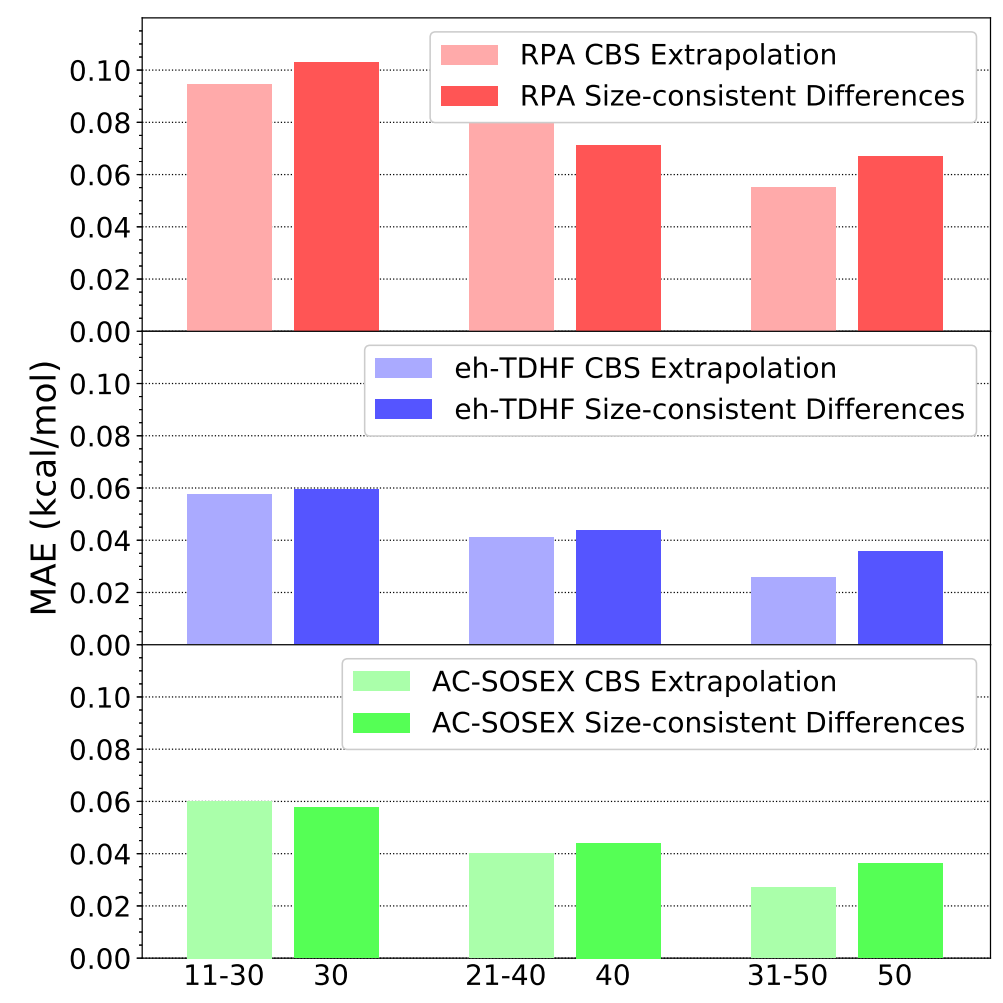

FIG. 3: Mean absolute errors (MAEs) of RPA (red), eh-TDHF (blue) and AC-SOSEX (green) for the A24 test set interaction energies using CBS extrapolations for $E^{C B S(11,30)}, E^{C B S(21,40)}$, and $E^{C B S(31,50)}$, and size-consistent energy differences for $E^{N_{a u x}=30}, E^{N_{a u x}=40}$, and $E^{N_{a u x}=50}$. The $E^{C B S(41,70)}$ interaction energy was used as a reference (see text).

results with size-consistent energy differences computed at $N_{\text {aux }} / N_{v}=30,40$, and 50 by using the procedure discussed in Sec. III. For convenience, we denote with $E_{A}^{C B S(n, m)}$ the CBS interaction energy computed by extrapolating 20 correlation energies between $N_{a u x}=n \times N_{v}$ and $m \times N_{v}$ at the $A$ level of theory ( $A=\mathrm{RPA}$, eh-TDHF, or AC-SOSEX); more specifically, Eq. 17 was used to obtain CBS correlation energies for each system (dimer and monomer) and these values were then used to compute correlation energies. Similarly, $E_{A}^{N_{a u x}=n N_{v}}$ corresponds to the non-extrapolated (namely based on size-consistent energy differences) interaction energy at the $A$ level of theory obtained with $N_{a u x}=n \times N_{v}$.

The top panel of Fig. 3 shows the mean absolute errors (MAE) with respect to $E_{\mathrm{RPA}}^{C B S(41,70)}$ for the RPA interaction energies obtained from the CBS for three different intervals, $E_{\mathrm{RPA}}^{C B S(11,30)}, E_{\mathrm{RPA}}^{C B S(21,40)}$, and $E_{\mathrm{RPA}}^{C B S(31,50)}$, and obtained from energy differences for three 
different values of $N_{\text {aux }}, E_{\mathrm{RPA}}^{N_{\text {aux }}=30}, E_{\mathrm{RPA}}^{N_{a u x}=40}$, and $E_{\mathrm{RPA}}^{N_{a u x}=50}$. In the middle and lower panels of Fig. 3, analogous results are shown for the eh-TDHF and AC-SOSEX methods respectively. The detailed results for each dimer in the the A24 test set are provided in the supporting information. From the three panels, we observe that the MAEs are rather small in all cases. However, considering the small energy scales involved in the A24 test set (the average interaction energy at the CCSDT(Q) level of theory is about $2 \mathrm{kcal} / \mathrm{mol}^{49}$ ), a high level of convergence is also particularly important. By considering Fig. 3 we can notice that: (1) RPA presents larger MAEs than the beyond-RPA methods, hinting that its convergence is slower and/or less stable; this empirically observed behavior might be related to a reduced cusp problem in beyond-RPA approximations, although further investigation will be necessary in the future for a better understanding; (2) if the maximum value for $N_{\text {aux }}$ is the same, results based on the CBS extrapolation or on the energy differences have similar accuracy; (3) MAEs systematically decrease by increasing the maximum value of $N_{\text {aux }}$; (4) the value of $N_{\text {aux }}=40 \times N_{v}$ used in our previous work ${ }^{16}$ to compute interaction energies provides a good level of accuracy, especially for the beyond-RPA methods.

According to this discussion for the A24 test set, the CBS results and the size-consistent energy differences have similar convergence rates and the two approaches are equivalent for most practical calculations. However, the size-consistent energy differences have the advantage of not being biased by the choice of a particular function to extrapolate to CBS.

\section{B. Methane-Formaldehyde Complex}

To further study the convergence rate of the RPA and beyond-RPA methods with respect to the auxiliary basis set size, we computed six points on the potential energy surface (PES) of the methane-formaldehyde complex (MFC). For these points accurate coupledcluster benchmark results have been recently published ${ }^{69}$. The numerical results for the MFC complex are also based on FHI pseudopotentials. This system allows us to verify the accuracy of the methods discussed in Sec. II and the reliability of the convergence techniques discussed in Sec. III also for the case of out-of-equilibrium geometries. As in the case of the A24 test set, we use $E^{C B S(41,70)}$ energies as the reference and we test the convergence of the CBS extrapolated values for three intervals, $E^{C B S(11,30)}, E^{C B S(21,40)}$, and $E^{C B S(31,50)}$, and the energy differences at three values of $N_{\text {aux }}, E_{\mathrm{RPA}}^{N_{a u x}=30 N_{v}}, E_{\mathrm{RPA}}^{N_{a u x}=40 N_{v}}$, and $E_{\mathrm{RPA}}^{N_{a u x}=50 N_{v}}$. In 
Fig. 4 we present the corresponding MAEs for the RPA, the eh-TDHF, and the AC-SOSEX. Detailed results are available in the supporting information. For the MFC, the MAEs decrease by increasing the value of $N_{\text {aux }}$, as was observed with the A24 test set. In contrast though, the convergence of energy differences significantly outperform CBS extrapolations for the beyond-RPA methods, whereas the converse is true for the RPA. To a certain extent this behavior can be interpreted by considering that size-consistent energy differences benefit from error cancellations, whereas the CBS extrapolations are sensitive to small fluctuations in the data. If for example we consider $E_{R P A}^{C B S(41,60)}$, which includes a subset of the points used for the reference $E_{R P A}^{C B S(41,70)}$, the MAE actually increases to $0.07 \mathrm{kcal} / \mathrm{mol}$, showing that small fluctuations influence the CBS extrapolation. On the other hand, the MAE on the energy differences continues to decrease with an increase in $N_{\text {aux }}$, reaching a value of 0.12 $\mathrm{kcal} / \mathrm{mol}$ for $E_{R P A}^{N_{a u x}=60 N_{v}}$. Thus, the convergence of the RPA is slower and more unstable, similar to that observed for the A24 test set. For the beyond-RPA methods, convergence is instead more systematic, with both approaches leading to similar results for sufficiently high values of $N_{\text {aux }}$. The method based on size-consistent energy differences is thus confirmed as a reliable and unbiased approach for the MFC as well, in particular, for the eh-TDHF and AC-SOSEX methods.

Fig. 5 shows the CBS results for the MFC PES compared to CCSD(T) reference values 69 . The RPA and beyond-RPA methods all capture the PES correctly, with the correct ordering in energy of the configurations. With respect to the coupled-cluster reference the mean absolute error for the RPA is $0.085 \mathrm{kcal} / \mathrm{mol}$. This value reduces to 0.061 and $0.066 \mathrm{kcal} / \mathrm{mol}$ for the eh-TDHF and AC-SOSEX respectively, highlighting the accurate nature of these methods. However, the convergence of RPA results is more problematic and, by considering the small energy scales involved in this PES, the eh-TDHF and AC-SOSEX results are certainly more reliable.

\section{Reaction energies}

After considering weakly bound systems, in this section we discuss results for the reaction energy test set by Hesselmann $\underline{70}$, which involves the breaking and formation of covalent bonds. While results for this test set were previously presented in Ref. 15, in this work we extend the auxiliary basis set up to $70 \times N_{v}$ and compare extrapolated results with energy dif- 


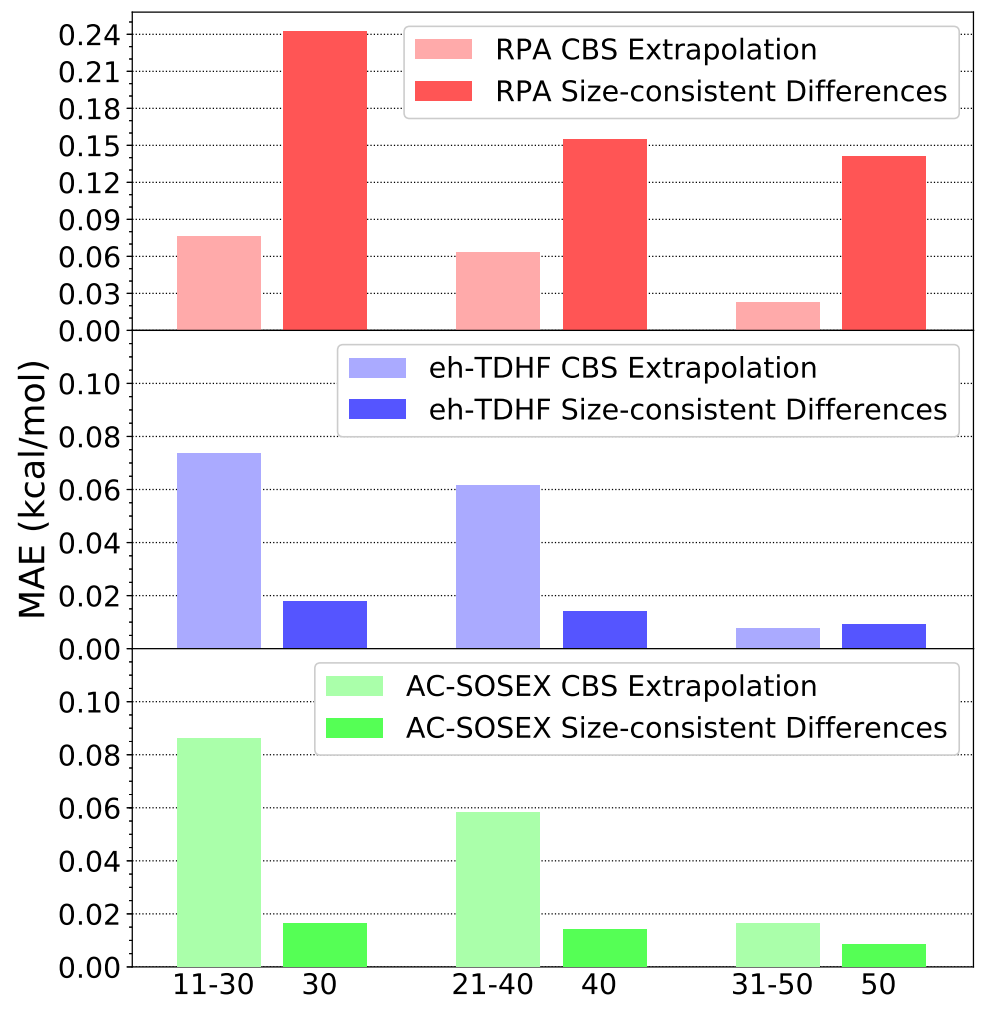

FIG. 4: Mean absolute errors (MAEs) of RPA (red), eh-TDHF (blue) and AC-SOSEX (green) for the methane-formaldehyde complex interaction energies using CBS extrapolations for $E^{C B S(11,30)}, E^{C B S(21,40)}$, and $E^{C B S(31,50)}$, and size-consistent energy differences for $E^{N_{a u x}=30}$, $E^{N_{a u x}=40}$, and $E^{N_{a u x}=50}$. The $E^{C B S(41,70)}$ interaction energy was used as a reference (see text).

ferences. Numerical results are based on norm-conserving pseudopotentials taken from the library of Schlipf and Gygi ${ }^{71}$, which has been generated within the optimized norm-conserving Vanderbilt pseudopotential scheme recently introduced by Hamann ${ }^{22}$ For applications involving covalent bonds the pseudopotentials have a sizeable effect on RPA and beyond-RPA correlation energies $\frac{15}{}$. This problem might be overcome in the future by using the projector augmented wave method. However, the values obtained in the PW/pseudopotential framework lead to errors with respect to coupled cluster reference results which are comparable to those of the Gaussian basis set; furthermore, the purpose of this paper is mainly to analyze the convergence behavior. Studying this test set is particularly interesting, since going from the reactants (e.g. $\mathrm{HCOOH}$ and $\mathrm{NH}_{3}$ ) to the products (e.g. $\mathrm{HCONH}_{2}$ and $\mathrm{H}_{2} \mathrm{O}$ ) involves the breaking and formation of covalent bonds. Accordingly, reactants and products could 


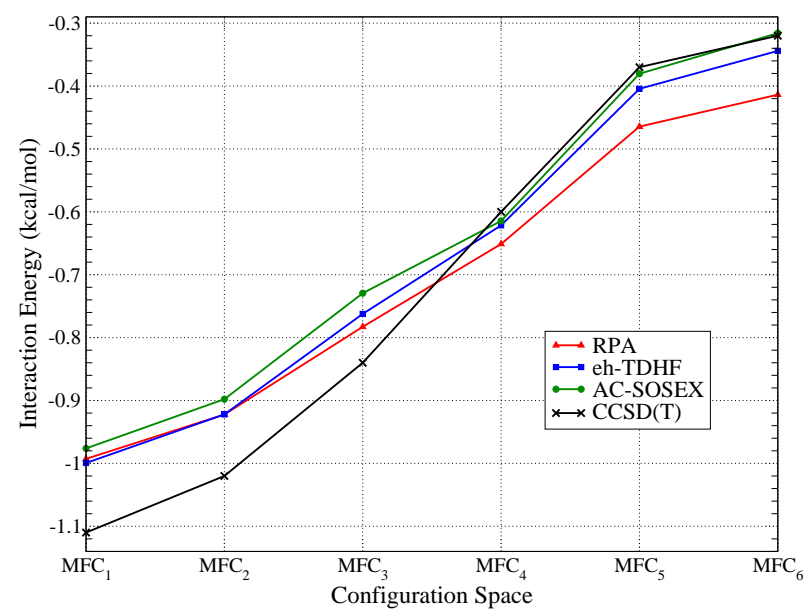

FIG. 5: Potential energy surface points $(\mathrm{kcal} / \mathrm{mol})$ of the methane-formaldehyde complex at the extrapolated RPA (red), eh-TDHF (blue) and AC-SOSEX (green) levels of theory compared with the $\operatorname{CCSD}(\mathrm{T})$ values (black).

have a rather different structure of the dielectric eigenspectrum and this could represent a challenge for the size-consistent energy difference method.

In Fig. 6] we present the MAEs obtained at the RPA, the eh-TDHF, and the AC-SOSEX levels of theory. As for the weakly bound systems, the MAEs are computed with respect to the $E^{C B S(41,70)}$ extrapolated value. Not surprisingly for this test set the deviation between CBS and size-consistent energy differences is more sizeable than for the weakly-interacting systems. A few observations are important for a full understanding of the results in Fig. 6; (1) As the average reaction energy at the CCSDT(Q) level of theory is about $29 \mathrm{kcal} / \mathrm{mol}$, the energy scales involved in this test set are usually larger than for weakly interacting systems and, accordingly, higher errors can be tolerate; (2) As mentioned before, the use of an $E^{C B S(41,70)}$ reference is to some extent arbitrary and creates a bias in favor of the CBS results; by using $E^{N_{a u x}=70}$ as a reference instead, the behavior shown in Fig. [6] would be swapped, with the size-consistent energy difference method showing a faster convergence; (3) The MAE of $E^{N_{a u x}=70}$ with respect to $E^{C B S(41,70)}$ (or vice versa) is within $0.09 \mathrm{kcal} / \mathrm{mol}$ for all the approximations and this observation point to the fact that the two methods studied in this work converge to the same values.

According to this discussion, the size-consistent energy difference method works well also beyond the expected range of applicability. The convergence of correlation energies for applications involving the breaking and formation of covalent bonds is more problematic 


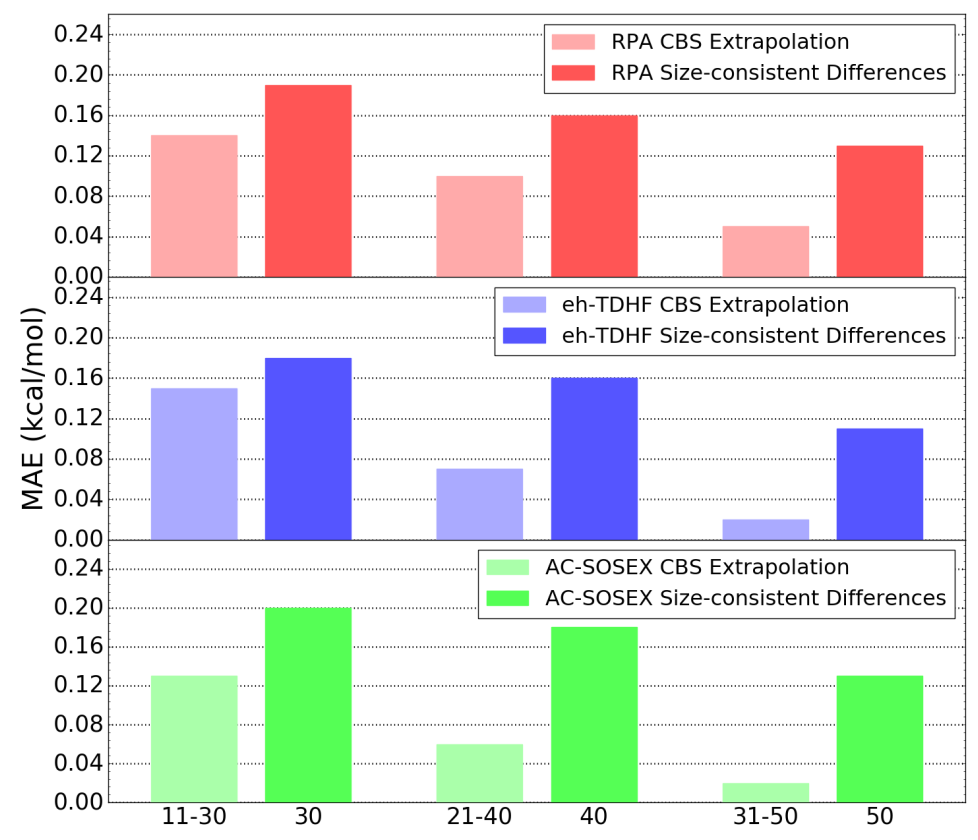

FIG. 6: Mean absolute errors (MAEs) of RPA (red), eh-TDHF (blue) and AC-SOSEX (green) for the reaction energy test set of Hesselmann $\frac{70}{-}$ using CBS extrapolations for $E^{C B S(11,30)}$, $E^{C B S(21,40)}$, and $E^{C B S(31,50)}$, and size-consistent energy differences for $E^{N_{a u x}=30}, E^{N_{a u x}=40}$, and $E^{N_{a u x}=50}$. The $E^{C B S(41,70)}$ interaction energy was used as a reference (see text).

but can achieve a good level of reliability.

\section{CONCLUSIONS}

In this work we analyzed the basis set convergence of the RPA method and of similar methods that include exchange effects, such as the eh-TDHF and AC-SOSEX. Specifically, two techniques were used to converge correlation energies: one based on a complete basis set extrapolation, and the other that converges energy differences by accounting for the size-consistency property. These two methods were tested by considering the A24 test set and six points on the potential energy surface of the methane-formaldehyde complex. It was found that both the CBS extrapolation and size-consistent energy differences converge to similar results at similar speeds as a function of the auxiliary basis set size. It was also shown that the RPA is in general characterized by a slower convergence than the beyondRPA methods. At least for the weakly bound systems considered in this paper, the two approaches have been found to be equivalent for practical purposes. If the breaking and 
formation of covalent bonds is involved, convergence is less trivial but satisfactory levels of accuracy can be reached.

The size-consistent energy difference technique represents a new and alternative scheme to conventional basis set extrapolation for converging results of correlated methods within a dielectric matrix formulation. While possibly less general than the CBS extrapolation, this approach has the advantage of not relying on any assumption on the model used for the fitting.

Although our present work focuses on molecular systems, the plane-wave basis set provides the numerical framework for most applications in solid state physics. For solids, the use of correlated methods for the ground-state correlation energy is much less common than in the quantum chemistry community due to challenges in methodology and implementation. Our work thus improves the understanding and extends the applicability of high-level methods for general systems, by pointing the way to more efficient implementation of RPAtype methods. The ideas discussed in this paper are not limited to RPA-type methods but can be extended to post-Hartree-Fock correlated methods too. Indeed, since the MP2 correlation energy can be reformulated within a dielectric matrix framework, the discussion in this paper can also be applied to this approach $\underline{67}$.

\section{ACKNOWLEDGEMENTS}

This work was supported by Agence Nationale de la Recherche under grant number ANR-15-CE29-0003-01. The results of this research have been achieved using GENCICCRT/CINES computational resources under grant x2017-085106 and the DECI resource ARCHER based in the United-Kingdom with support from the PRACE aisbl. The authors acknowledge financial support from the CNRS PICS program 2DvdW and from the French PIA project "Lorraine Université d'Excellence".

* dixit1@univ-lorraine.fr

† dario.rocca@univ-lorraine.fr

1 P. Hohenberg and W. Kohn, Phys. Rev. 136, B864 (1964).

2 W. Kohn and L. Sham, Phys. Rev. 140, A1133 (1965). 
3 J. P. Perdew and A. Zunger, Phys. Rev. B 23, 5048 (1981).

4 J. P. Perdew, K. Burke, and M. Ernzerhof, Phys. Rev. Lett. 77, 3865 (1996).

5 D. C. Langreth and J. P. Perdew, Solid State Comm. 17, 1425 (1975).

6 D. C. Langreth and J. P. Perdew, Phys. Rev. B 15, 2884 (1977).

7 M. Fuchs and X. Gonze, Phys. Rev. B 65, 235109 (2002).

8 J. Toulouse, I. C. Gerber, G. Jansen, A. Savin, and J. G. Ángyán, Phys. Rev. Lett. 102, 096404 (2009).

9 H.-V. Nguyen and S. de Gironcoli, Phys. Rev. B 79, 205114 (2009).

10 W. Zhu, J. Toulouse, A. Savin, and J. G. Ángyán, J. Chem. Phys. 132, 244108 (2010).

11 H.-V. Nguyen and G. Galli, J. Chem. Phys. 132, 044109 (2010).

12 X. Ren, P. Rinke, V. Blum, J. Wieferink, A. Tkatchenko, A. Sanfilippo, K. Reuter, and M. Scheffler, New J. Phys. 14, 053020 (2012).

13 D. Rocca, J. Chem. Phys. 140, 18A501 (2014).

14 B. Mussard, D. Rocca, G. Jansen, and J. G. Ángyán, J. Chem. Theory Comput. 12, 2191 (2016).

15 A. Dixit, J. G. Ángyán, and D. Rocca, J. Chem. Phys. 145, 104105 (2016).

16 A. Dixit, J. Claudot, S. Lebègue, and D. Rocca, J. Chem. Theory Comput. 13, 5432 (2017).

17 A. Marini, P. García-González, and A. Rubio, Phys. Rev. Lett. 96, 136404 (2006).

18 S. Lebègue, J. Harl, T. Gould, J. G. Ángyán, G. Kresse, and J. F. Dobson, Phys. Rev. Lett. 105, $196401(2010)$.

19 T. Björkman, A. Gulans, A. V. Kraheninnikov, and R. M. Nieminem, Phys. Rev. Lett. 108, $235502(2012)$.

20 A. Gulans, J. Chem. Phys. 141, 164127 (2014).

21 J. Harl and G. Kresse, Phys. Rev. B 77, 045136 (2008).

22 D. Lu, Y. Li, D. Rocca, and G. Galli, Phys. Rev. Lett. 102, 206411 (2009).

23 J. Harl, L. Schimka, and G. Kresse, Phys. Rev. B 81, 115126 (2010).

24 F. Kaoui and D. Rocca, J. Phys.: Condens. Matter 28, 035201 (2016).

25 M. M. J. Grundei and A. M. Burow, J. Chem. Theory Comput. 13, 1159 (2017).

26 X. Ren, P. Rinke, and M. Scheffler, Phys. Rev. B 80, 045402 (2009).

27 S. Kurth and J. P. Perdew, Phys. Rev. B 59, 10461 (1999).

28 F. Furche, Phys. Rev. B 64, 195120 (2001). 
29 N. L. Nguyen, N. Colonna, and S. de Gironcoli, Phys. Rev. B 90, 045138 (2014).

30 A. Grüneis, M. Marsman, J. Harl, L. Schimka, and G. Kresse, J. Chem. Phys. 131, 154115 (2009).

31 J. G. Ángyán, R.-F. Liu, J. Toulouse, and G. Jansen, J. Chem. Theory Comput. 7, 3116 (2011).

32 X. Ren, A. Tkatchenko, P. Rinke, and M. Scheffler, Phys. Rev. Lett. 106, 153003 (2011).

33 T. Olsen and K. S. Thygesen, Phys. Rev. B 86, 081103 (2012).

34 D. Lu, J. Chem. Phys. 140, 18A520 (2014).

35 F. Furche and T. van Voorhis, J. Chem. Phys. 122, 164106 (2005).

36 A. Heßelmann and A. Görling, Phys. Rev. Lett. 106, 093001 (2011).

37 N. Colonna, M. Hellgren, and S. de Gironcoli, Phys. Rev. B 90, 125150 (2014).

38 A. D. McLachlan and M. A. Ball, Rev. Mod. Phys. 36, 844 (1964).

39 J. E. Bates and F. Furche, J. Chem. Phys. 139, 171103 (2013).

40 S. Grimme, J. Comp. Chem. 25, 1463 (2004).

41 A. Tkatchenko and M. Scheffler, Phys. Rev. Lett. 102, 073005 (2009).

42 A. Tkatchenko, R. A. Di Stasio, R. Car, and M. Scheffler, Phys. Rev. Lett. 108, 236402 (2012).

43 M. Dion, H. Rydberg, E. Schroder, D. C. Langreth, and B. I. Lundqvist, Phys. Rev. Lett. 92, $246401(2004)$.

44 O. A. Vydrov and T. R. Voorhis, J. Chem. Phys. 133, 244103 (2010).

45 C. Schwartz, in Methods in Computational Physics, Vol. 2, edited by B. Alder, S. Fernbach, and M. Rotenberg (Academic Press, 1963) p. 241.

46 T. Helgaker, W. Klopper, H. Koch, and J. Noga, J. Chem. Phys. 106, 9639 (1997).

47 A. Halkier, T. Helgaker, P. Jørgensen, W. Klopper, H. Koch, J. Olsen, and A. K. Wilson, Chem. Phys. Lett. 286, 243 (1998).

48 D. Feller, K. A. Peterson, and T. D. Crawford, J. Chem. Phys. 124, 054107 (2006).

49 J. Řezáč and P. Hobza, J. Chem. Theory Comput. 9, 2151 (2013).

50 J. Řezáč and P. Hobza, Chem. Rev. 116, 5038 (2016).

51 C. Møller and M. S. Plesset, Phys. Rev. 46, 618 (1934).

52 R. J. Bartlett and M. Musiał, Rev. Mod. Phys. 79, 291 (2007).

53 W. Kutzelnigg and J. D. Morgan III, J. Chem. Phys. 96, 4484 (1992).

54 H. F. Wilson, F. Gygi, and G. Galli, Phys. Rev. B 78, 113303 (2008).

55 H.-V. Nguyen, T. A. Pham, D. Rocca, and G. Galli, Phys. Rev. B 85, 081101 (2012). 
56 T. A. Pham, H.-V. Nguyen, D. Rocca, and G. Galli, Phys. Rev. B 87, 155148 (2013).

57 Y. Ping, D. Rocca, and G. Galli, Chem. Soc. Rev. 42, 2437 (2013).

58 M. Govoni and G. Galli, J. Chem. Theory Comput. 11, 2680 (2015).

59 D. Lu, H.-V. Nguyen, and G. Galli, J. Chem. Phys. 133, 154110 (2010).

60 S. Baroni, S. de Gironcoli, A. Dal Corso, and P. Giannozzi, Rev. Mod. Phys. 73, 515 (2001).

61 B. Walker, A. M. Saitta, R. Gebauer, and S. Baroni, Phys. Rev. Lett. 96, 113001 (2006).

62 D. Rocca, R. Gebauer, Y. Saad, and S. Baroni, J. Chem. Phys. 128, 154105 (2008).

63 D. Rocca, D. Lu, and G. Galli, J. Chem. Phys 133, 164109 (2010).

64 P. Umari, G. Stenuit, and S. Baroni, Phys. Rev. B 81, 115104 (2010).

65 P. Giannozzi, S. Baroni, N. Bonini, M. Calandra, R. Car, C. Cavazzoni, D. Ceresoli, G. L. Chiarotti, M. Cococcioni, I. Dabo, A. Dal Corso, S. de Gironcoli, S. Fabris, G. Fratesi, R. Gebauer, U. Gerstmann, C. Gougoussis, A. Kokalj, M. Lazzeri, L. Martin-Samos, N. Marzari, F. Mauri, R. Mazzarello, S. Paolini, A. Pasquarello, L. Paulatto, C. Sbraccia, S. Scandolo, G. Sclauzero, A. P. Seitsonen, A. Smogunov, P. Umari, and R. M. Wentzcovitch, J. Phys.: Condens. Matter 21, 395502 (2009).

66 P. Giannozzi, O. Andreussi, T. Brumme, O. Bunau, M. B. Nardelli, M. Calandra, R. Car, C. Cavazzoni, D. Ceresoli, M. Cococcioni, N. Colonna, I. Carnimeo, A. Dal Corso, S. de Gironcoli, P. Delugas, R. A. DiStasio Jr., A. Ferretti, A. Floris, G. Fratesi, G. Fugallo, R. Gebauer, U. Gerstmann, F. Giustino, T. Gorni, J. Jia, M. Kawamura, H.-Y. Ko, A. Kokalj, E. Küçükbenli, M. Lazzeri, M. Marsili, N. Marzari, F. Mauri, N. L. Nguyen, H.-V. Nguyen, A. Otero-de-laRoza, L. Paulatto, S. Poncé, D. Rocca, R. Sabatini, B. Santra, M. Schlipf, A. P. Seitsonen, A. Smogunov, I. Timrov, T. Thonhauser, P. Umari, N. Vast, X. Wu, and S. Baroni, J. Phys.: Condens. Matter 29, 465901 (2017).

67 A. Dixit, J. Claudot, S. Lebègue, and D. Rocca, J. Chem. Phys. 146, 211102 (2017).

68 T. Gould, J. Toulouse, J. G. Ángyán, and J. F. Dobson, J. Chem. Theory Comput. 13, 5829 (2017).

69 K. B. Moore, K. Sadeghian, C. D. Sherrill, C. Ochsenfeld, and H. F. Schaefer, J. Chem. Theory Comput. 13, 5379 (2017).

70 A. Heßelmann, Phys. Rev. A 85, 012517 (2012).

71 M. Schlipf and F. Gygi, Computer Physics Communications 196, 36 (2015).

72 D. Hamann, Physical Review B 88, 085117 (2013). 